\title{
Epilepsie im Kriege ${ }^{1}$ ).
}

\author{
Von \\ Dr. J. Leva. \\ (Eingegangen am \%. November 1921).
}

Der klinische Begriff der Epilepsie hat im Laufe der Jahre eine zunehmend engere Umgrenzung und schärfere Bestimmung erfahren. Die große Gruppe der Krankheitszustände, die ohne äußere Ursache mit Anfällen von Krämpfen und Bewußtlosigkeit einhergehen, bei denen es zu mannigfachen Verletzungen, zu vorübergehenden Lähmungen von Blase und Mastdarm, sowie zu zeitweisen Störungen der Bewußtseinstätigkeit kommen kann, darf heute nicht mehr schlechtweg Epilepsie genannt werden. Mag auch der Krampfanfall als Hauptsymptom der Epilepsie gelten, er ist nicht mehr das einzige und in jedem Falle epilepsiebeweisende Krankheitszeichen. Erst dann, wenn mit einer gewissen regelmäßigen Wiederholung Anfälle oder sog. epileptisch-äquivalente Zustände auftreten, wenn sich im weiteren Verlauf ein geistiger Schwächezustand entwickelt, können wir mit Recht annehmen, daß ein fortschreitendes organisches Gehirnleiden vorliegt und die Diagnose auf Epilepsie stellen.

Ganz ähnlich wie die Epilepsie kann eine Gruppe von anderen Gehirnleiden mit einem zeitweisen Auftreten von Krampfanfällen einhergehen; so die Urämie, die Bleivergiftung, die progressive Paralyse und auch Hirntumoren. Die Verfeinerung der diagnostischen Hilfsmittel und die klinische Forschung haben uns gelehrt, diese Krankheitsformen von der Epilepsie zu unterscheiden. Während uns bei dieser die Entstehung und das Auftreten der Krampfanfälle bis jetzt immer noch unklar geblieben ist, können wir dort mit genügend Recht annehmen, daß die Anfälle durch chemische oder anatomische Reize der Gehirnsubstanz ausgelöst werden. Als Bestandteile des Grundleidens verschwinden

1) Die Arbeit wurde während meiner Tätigkeit als ordinierender Arzt am Festungslazarett XXVII zu Straßburg i. Els. (Chefarzt Geheimer Medizinalrat Prof. Dr. Wollenberg) verfaßt. Die Veröffentlichung während des Krieges mußte unterbleiben auf Grund eines Verbotes der militärischen Zensurstelle. Sie erfolgt jetzt in abgeänderter Form. 
sie wieder, wie sie gekommen sind, wenn dieses sich bessert. Wir sprechen von symptomatischer Epilepsie. Weitere Krankheitsformen, bei denen bisweilen epileptische Anfälle beobachtet werden, ohne daß Epilepsie im eigentlichen Sinne vorliegt, sind in den letzten Jahren mehr und mehr bekannt geworden. Es sind dies gewisse Fälle von Neuro- und Psychopathie. Westphal lenkte als erster im Jahre 1871 die Aufmerksamkeit auf derartige Krankheitsformen. Er beobachtete ein Auftreten von epileptischen Anfällen bei Kranken, die an Platzangst litten. Auf Grund. seiner damaligen Erfahrungen kam bereits Westphal zum Schlusse, „daß derartige epileptoide Anfälle zu den allerhäufigsten Symptomen der verschiedenartigsten psychopathischen und neuropathischen $\mathrm{Zu}$ stände gehören." In der Folgezeit wurden ähnliche Beobachtungen von Oppenheim und von Bratz mitgeteilt. Oppenheim beschrieb epileptische Anfälle bei Individuen, die teils an Symptomen von reizbarer Schwäche bei typischer Neurasthenie, teils an Angstzuständen, Phobien, Tics oder vasomotorischen Störungen litten, Zustände, wie sie unter dem Begriff Psychasthenie zusammengefaßt werden. In allen diesen Fällen konnten Gelegenheitsursachen, wie heftige Gemütsbewegungen, körperliche oder geistige Überanstrengung oder auch Exzesse zu dem Auftreten der Anfülle führen. Oppenheim spricht hier von psychasthenischen Anfällen. Bratz konnte diese Beobachtungen Oppenheims in noch weitgehenderem Maße bestätigen. Außer bei den Neurasthenikern und. Psychasthenikern sah er ein Auftreten von epileptischen Anfällen auch bei gewissen Formen von Psychopathie, insbesondere häufig bei der Gruppe der unstet Degenerierten. Bratz beobachtete Anfälle der verschiedensten Art: Außer den typisch epileptischen Schwindel- and Ohnmachtsanfällen, Schlaf- und Verwirrtheitszustände, ferner psychische Anfälle, die den epileptischen Äquivalenten entsprachen. Alle diese letzterwähnten Krankheitsformen bei Neuro- und Psychopathen (Westphal, Oppenheim, Bratz) unterscheiden sich grundsätzlich von der Epilepsie durch zwei Merkmale: Die Anfälle treten nur episodenhaft auf; sie führen nicht zur Verblödung. Bratz spricht ganz allgemein von affektepileptischen Anfällen und will unter diesen auch die psychasthenischen Formen (Oppenheim) verstanden wissen.

Eine weitere Gruppe von epilepsieartigen Erkrankungen, die besonders im kindlichen Alter auftreten, sind unter der Bezeichnung narcoleptische Anfälle bekannt geworden. Thre klinische Bedeutung ist noch nicht ganz sicher gestellt.

Die Erkennung der typischen Epilepsie war auch im Kriege nicht schwierig. Sie stützte sich in erster Linie auf die Krampfanfälle. Mochten auch bisweilen andersartige (Äquivalente) Anzeichen zuerst auf das Leiden hindeuten, so waren es doch ganz vorzugsweise die Anfälle, die 
am weitaus häufigsten und untrüglichsten die Epilepsie feststellen ließen. Es liegt in der Natur der Krankheit begründet, daß sie sich ganz vorzugsweise hierdurch äußert. Diese Tatsache kommt dem auf subtile klinische Beobachtungen meist nicht vorbereiteten Truppenarzt besonders zustatten.

Die meist vollkommene oder sehr tiefe BewuBtlosigkeit, die Einfachheit der klonisch-tonischen Muskelkrämpfe, die Cyanose des Gesichts, die oft vorkommenden Zungenbisse oder sonstigen Verletzungen, das Versagen von Blase und Mastdarm im Anfall, die verhältnismäßige Kürze der Dauer: alle diese Merkmale sind im Gegensatz zum hysterischen dem epileptischen Anfall besonders eigentümlich. Erweisen sich weiterhin im Anfall die Pupillen als lichtstarr, ist das Babinskische Fußsohlenphänomen positiv, oder sind noch sonstige Anzeichen des organischen Komas vorhanden, so ist nur in den seltensten Fällen ein Zweifel an der Echtheit der epileptischen Natur berechtigt. Erfahren wir sodann nach Wiederkehr des Bewußtseins vom Kranken selbst, daß er schon in früheren Jahren an Anfällen ähnlicher Art gelitten hat, so bestätigt sich der Verdacht mehr und mehr, daß ein chronisches Krampfleiden vorliegt. Aber nicht in allen Fällen gelingt die Diagnose so leicht und mühelos. Die große Vielgestaltigkeit der einzelnen Beobachtungen, aus denen sich der Anfall zusammensetzt, läßt über seine Natur oft im Unklaren. In wieder anderen Fällen wurde der Anfall nieht von ärztlicher Seite beobachtet, so daß auch hierdurch Zweifel an seiner Art wohl berechtigt waren. Auch die sonstigen Begleitumstände, die dem Anfall vorausgingen, schienen nicht selten seine epileptische Natur in Frage zu stellen.

So konnte bei dem Vorausgehen von psychischen Erregungen, Schreck oder sonstiger Emotion mit Recht manchmal an Hysterie gedacht werden; in anderen Fällen waren körperliche Strapazen oft unter Mitwirkung von Hitze, Hunger und Durst vorausgegangen; hier war der Verdacht, daß plötzliche Herzschwäche oder ein Versagen des Vasomotorensystems (Hitzschlag) dem anfallsartigen Zustande zugrunde lag, wohl berechtigt.

Es ergibt sich hieraus, daß es nach der Fntstehung und Verlaufsweise des einzelnen Anfalls oft schlechterdings unmöglich war, seine epileptische Natur und damit das Leiden selbst einwandfrei festzustellen.

Nur eine genaue Beobachtung des Kranken, auch in der anfallsfreien Zeit, die Gesamtberücksichtigung aller objektiven Einzelbeobachtungen zusammen mit den subjektiven Angaben des Kranken und zeugenmäßigen Erhebungen über das Vorleben ermöglichten eine endgültige Diagnose in der Regel auch bei anfänglich unklaren Fällen. 
I.

Die Beobachtungen, die dieser Arbeit zugrunde liegen, beziehen sich auf 188 Fälle. Bei 152 hatte schon Epilepsie vor dem Kriege bestanden. Bei 36 wurden Anfälle zuerst im Kriege beobachtet. Wenden wir uns zunächst den Fällen zu, bei denen schon vor dem Kriege die sicheren Zeichen der Epilepsie bestanden hatten. Wie bereits erwähnt, war bei der Einstellung von vielen ein ärztlicher Nachweis des früheren Leidens nicht erbracht worden. Bei anderen waren die Anfälle in den letzten Jahren so selten aufgetreten, daß, wie bei der Leichtgläubigkeit derartig Kranker wohl verständlich, an Heilung gedacht werden konnte. Wieder anderen war die Natur und Ernsthaftigkeit des Leidens nicht klar geworden; sie wußten wohl von gelegentlich auftretenden Anfällen, gaben sich aber keine weitere Rechenschaft über ihren Krankheitsaustand. So kam es, daß sie sich dem Kriegsdienste und seinen Strapazen unterzogen, bis die neu auftretenden Anfälle ihnen und den Truppenärzten zu erkennen gaben, daß das Leiden noch fortbestand. Von diesen 152 Fällen hatten $55(36,2 \%)$ im Frieden aktiv gedient.

Bei 22 Fällen waren epileptische Anfälle vor der Dienstzeit aufgetreten.

Bei 7 Fällen waren epileptische Anfälle zuerst während der Dienstzeit aufgetreten.

Bei 26 Fällen waren epileptische Anfälle zuerst nach der Dienstzeit aufgetreten.

Die letztgenannten Fälle gehören der typischen Spätepilepsie an. Die übrigen 29 sind im Rahmen dieser Betrachtung besonders interessant. Von den schon vor der Dienstzeit epileptischen 22 Fällen waren bei 12 auch während der aktiven Dienstzeit Anfälle aufgetreten. Aber sie waren offenbar so selten und wenig erheblicher Natur, daß ihnen keine besondere Bedeutung beigemessen wurde. Andernfalls wäre die Entlassung vom Dienste unvermeidlich geworden. Bei 3 anderen Fällen trat während des aktiven Dienstes eine zunehmende Verschlimmerung ein; sie wurden als dienstunbrauchbar entlassen. Bei den übrigen 7 Fällen waren, wie die Kranken selbst glaubhaft versichert hatten, die Anfälle während des aktiven Dienstes ausgeblieben, nachdem sie vorher wiederholt aufgetreten waren.

Bei den übrigen 97 Fällen, die nicht gedient hatten, war die Krankheit in einem großen Teil der Fälle vor dem 20. Jahre aufgetreten und hatte somit die Dienstunbrauchbarkeit verursacht; wieder andere waren aus nicht mehr feststellbaren anderen Gründen militärfrei geworden; bei ihnen entwickelte sich die Epilepsie erst im 2. und 3. Dezennium.

Bei der ätiologischen Forschung der Epilepsie hat man zu unterscheiden:

1. Ursachen; die zur Krankheitsanlage führen (Hereditäre Anlage, sonstige äußere oder uns unbekannte innere Vorgänge). 
2. Ursachen, die die Krankheit nach bereits entwickelter Anlage auslösen und offenbar werden lassen.

Derartig auslösende Ursachen haben bei der Epilepsie eine besondere Bedeutung.

Die Gruppierung unserer 152 Fälle nach Altersgrenzen war wie folgt:

\begin{tabular}{c||c|c|c|c|c|c|c|c|c}
\hline Jahre & $0-10$ & $10-15$ & $15-20$ & $20-25$ & $25-30$ & $30-35$ & $35-40$ & 40 u. mehr \\
\hline Fälle & 38 & 20 & 28 & 33 & 16 & 11 & 6 & 0 \\
$\% / 0$ & 25 & 13 & 18 & 22 & 11 & 7 & 4 & -
\end{tabular}

Hiernach ergibt sich ein ganz vorzugsweise erstmaliges Auftreten des Leidens in den Jahren der ersten Kindheit. Aber auch innerhalb des ganzen 1. und 2. Dezenniums tritt die Krankheit besonders auf und erst im 3. erkennen wir ein Nachlassen der Krankheitsbereitschaft. Es erkrankten so bis zum 2. Dezennium $56 \%$, bis zum $3.88 \%$ unserer Fälle. Mag auch unser Material in gewisser Hinsicht besonders geartet und einseitig genannt werden, es bestätigt sich doch auch hier wieder die Beobachtung, die an weit größerer Krankenzahl von zahlreichen Autoren gemacht wurde.

Es fanden bis zum 10. Jahre:

Binswanger bis zum 10 . Jahre 35,6 ; bis zum 20. Jahre $41,6 \%$, Bergex " " $10 . \quad$ " 41,$3 ; \quad$ " "20. " $24,9 \%$, Cowers " " $\quad 10 . \quad, 31 ; ", \quad$ " $20 . \quad$ " $45,8 \%$, Finkh $\quad$ " " $10 . \quad, \quad 40,5 ; \quad, \quad$, $20 . \quad, \quad 38,7 \%$.

Diese Zahlen lassen die hohe Bedeutung der ersten 2 Dezennien für das Auftreten der Krankheit erkennen. Bei unseren 152 Fällen traten die ersten Anfälle 86 mal innerhalb der beiden ersten Dezennien, 66 mal innerhalb der ersten 3 Dezennien auf. Hiernach besteht also auch während des 3. Jahrzehnts noch eine ausgesprochene Reigung zum Auftreten des Leidens. Diese Tatsache steht im Widerspruch zu den Statistiken anderer Untersucher und wir können mit Recht fragen:

Liegt diese Besonderheit in der Art unseres gesamten Beobachtungsmaterials begründet oder hatten hier besondere Schädlichkeiten, die noch vorwiegend das 3. Dezennium betreffen, mit anderen Ursachen gleichzeitig zusammengewirkt?

Die Frage einer besonderen Auslese darf mit Recht bejaht werden. Es ist wohl anzunehmen, daß die durchschnittliche Beschaffenheit der Epileptiker, die im Kriege dem Arzt zur Beobachtung kamen, sich nach Art und Schwere der. Krankheit von jenen wahllosenTypen der Epilepsie unterscheidet, die in Kliniken und. Epileptikeranstalten gesammelt zur Beobachtung kommen. Dort finden wir vorwiegend Kranke mit seltenen Anfällen, bei denen die Begleit- und Folgeerscheinungen des Leidens 
noch nicht zu tiefgreifenden Veränderungen der Persönlichkeit geführt haben, hier wohl in der Mehrzahl der Fälle Individuen, die infolge steten Fortschreitens der Krankheit der geistigen Schwäohe und dem Siechtum bereits verfallen sind. Eine weitere Klärung erfährt die Frage, wenn wir aus der oben stehenden Altersstatistik die Fälle absondern, die im Frieden gedient haben. Wir erhalten hiernach die folgende Übersicht:

\begin{tabular}{c||c|c|c|c|c|c|c|c}
\hline \hline Jahre & $0-10$ & $\mathbf{1 0 - 1 5}$ & $15-20$ & $\mathbf{2 0 - 2 5}$ & $\mathbf{2 5 - 3 0}$ & $\mathbf{3 0 - 3 5}$ & $\mathbf{3 5 - 4 0}$ & $\mathbf{4 0}$ u. mehr \\
\hline Fälle & 11 & 4 & 5 & 18 & 8 & 7 & 2 & - \\
$\%$ & 20 & 7,3 & 9,1 & 32,7 & 14,5 & 12,7 & 3,7 & -
\end{tabular}

Es entfallen hiernach bei der Gruppe der Epileptiker, die in Friedenszeiten gedient hatten, auf das 3 . Dezennium $46 \%$ auf das 1 . und 2. zusammen $35,7 \%$.

Die Alterstabelle der nicht gedienten Epileptiker berechnet sich durch Subtraktion wie folgt:

\begin{tabular}{c||c|c|c|c|c|c|c|c}
\hline \hline Jahre & $0-10$ & $10-15$ & $15-20$ & $20-25$ & $25-30$ & $30-35$ & $35-40$ & 40 u. mehr \\
\hline \hline Fälle & 27 & 16 & 23 & 15 & 8 & 4 & 4 & - \\
$\%$ & 27,8 & 16,5 & 23,7 & 15,4 & 8,2 & 4,2 & 4,2 & -
\end{tabular}

Nach dieser Gegenüberstellung ist also das Auftreten der Epilepsie bei Individuen mit aktiver Dienstzeit im 3. Dezennium noch besonders häufig. Ob dieser Umstand auf den militärischen Dienst als solchen zurückzuführen ist, erscheint nach den früheren Ausführungen zweifelhaft. Was für Gründe sonst sich dafür anführen lassen, bleibt einstweilen eine offene Frage.

Bei unseren 152 Fällen wurde $68 \mathrm{mal}(=44,7 \%)$ erbliche Belastung angegeben. Nach dem Vorschlage der genaueren Einteilung nach Kraepelin zerfiel diese Gesamtbelastung wieder in:

30 Fälle $(19,7 \%)$ von Krampfanfällen in der Ascendenz und Seitenverwandtschaft.

34 Fälle $(22,6 \%)$ von sonstigen Nervenkrankheiten und Psychosen.

4 Fälle $(2,6 \%)$ von Linkshändigkeit.

Nach diesen Beobachtungen besteht also eine beträchtliche Neigung der Epilepsie zu gleichförmiger Vererbung. In 14 Fällen bestand Alkoholismus; in 5 Fällen bestand hereditäre Belastung und Alkoholismus zusammen.

Bemerkesnwert war weiterhin das Vorkommen von Traumen in der Vorgeschichte unserer Epileptiker. Derartige Angaben fanden sich in 32 Fällen $(21 \%)$. In 16 Fällen schlob sich das Leiden unmittelbar an das Trauma an, in den übrigen 16 war ein mehr oder weniger langer Zeitraum 
zwischen Trauma und erstem epileptischem Anfall verstrichen. Die Pause zwischen beiden betrug nur wenige Tage bei 9 Füllen; das längste Zeitintervall war 16 Jahre. Die durchschnittliche Dauer zwischen Trauma und Epilepsie berechnete sich auf 2,1 Jahre. In 2 Fällen war der erste Anfall nach einem kalten Bade aufgetreten.

Ửber die ursächliche Bedeutung, die den Kopftraumen im allgemeinen zukommt, sind die Ansichten verschieden. Finzelne schreiben ihm nur die Rolle einer Gelegenheitsursache zu für das schon latent vorhandene Leiden; das Trauma erzeuge schlimmstenfalls nur die Prädisposition. Die Sicherheit eines bestehenden Zusammenhangs zwischen Trauma und Epilepsie wird zunehmend problematischer, wenn beide durch einen Zeitraum von mehreren Jahren voneinander getrennt sind. Kraepeli n hält den Zusammenhang für eine Reihe von Fällen für zweifellos, glaubt aber doch auch, daß dieser namentlich bei leichteren Anfällen nur selten nachweisbar sein wird. Jedenfalls spielt die Schwere der Verletzung eine wesentliche Rolle. Genauere Anhaltspunkte lassen sich bei dem jetzigen Material nur schwer erbringen. Man wird nicht selten neben den Traumen noch eine Reihe von anderen Ursachen zu berücksichtigen haben, die für die Entstehung des Leidens weiterhin verantwortlich zu machen sind. Unser Material zerfällt sonach in Fälle, bei denen nur ein Trauma allein, und in solche, bei denen noch weitere auxiliare Ursachen (hereditäre Belastung, körperliche Krankheiten, Alkohol) angegeben wurden.

Reine Fälle hatten 26 mal vorgelegen. Fälle mit auxiliaren Ursachen 6 mal.

Eine uns heute ganz vorzugsweise interessierende Frage lautet: Inwieweit wirlst der Krieg auf bereits bestehende Epilepsie verschlimmernd? Haben wir ein Recht, anzunehmen, daß die Verlaufsweise und Schwere des Leidens durch die Teilnahme am Kriege und seine mannigfachen Schädigungen eine Änderung erfahren? Bei der Beantwortung haben wir auf zweierlei wesentliche Dinge zu achten:

1. auf die Dauer und besondere Art des Kriegsdienstes;

2. auf die Verlaufsweise der Epilepsie, d. h. etwaige Änderungen, die seit dem Kriegsdienste eingetreten sind.

Die Dauer und Art der Kriegsteilnahme ist ohne weiteres von den Patienten zu erfahren. Für eine Beurteilung der Verlaufsweise des Leidens gibt uns die Häufigkeit der Anfälle einen brauchbaren Anhaltspunkt. Dort, wo sie häufiger und in zunehmender Schwere auftreten, werden wir wohl annehmen können, daß durch die Kriegsteilnahme eine Verschlimmerung eingetreten ist; aber auch dann können wir für viele Fälle zumeist keine bestimmte Erklärung geben, durch welche besondere Art von Schädigung diese Verschlimmerung bedingt ist. Für zahlreiche andere Fälle geben uns die glaubhaften Angaben der Erkrankten, die 
sich oft leicht durch Zeugen bestätigen lassen, weitere Fingerzeige. Wir erfahren oft, daß während des anstrengenden Dienstes im Felde, beim Schanzen, auf dem Marsche, nach längeren Gefechten oder auch nach fortgesetzter Artilleriebeschießung das frühere Leiden plötzlich in erneuter Heftigkeit wieder auftrat.

Häufig sind es Anstrengungen und zunehmende körperliche Sehwächezustände, die zur Vermehrung und größeren Heftigkeit der Anfälle führen. Bei einer Anzahl von Kriegsteilnehmern war allerdings ein Wechsel in der Verlaufsweise des Leidens schon kurze Zeit nach der Einstellung eingetreten. Hier wird von einer eigentlichen Kriegsschädigung nicht wohl gesprochen werden können. Die Änderung in der Lebensweise und sonstige äußere Bedingungen mit den auch nicht selten hinzukommenden gemütlichen Erregungen werden im wesentlichen diesen Unterschied bedingen. Die Bereitschaft zur anfallsartigen Entladung ist bei derartigen Epileptikern eine besonders große.

Anders wieder bei einer anderen Gruppe von Fällen. Sie hatten die Ausbildung ohne besondere Störung überstanden, waren auch längere Zeit, bis zu mehreren Monaten, im Felde und unterzogen sich dem vollen Dienste, bis schließlich ein Anfall auftrat. Dieser Wechsel im Verlaufstypus des Leidens erinnert uns an die obenerwähnten Fälle, bei denen bereits von Kindheit an bestehende Anfälle während der aktiven Militärzeit überhaupt ausgeblieben waren. Die Versuchung liegt nahe, für den anfangs günstigen Verlauf dieser Fälle im jetzigen Kriege den Wechsel in der Lebensweise und andere äußere Bedingungen anzuschuldigen.

Zunehmende Anstrengungen und gehäufte Kriegsschäden führten aber doch schließlich zu einer ungünstigen Wendung des Leidens. Kriegsdienstbeschädigung kann man aber nach diesen Ausführungen bei alten Epileptikern in der Regel nicht annehmen.

II.

Ein besonderes Interesse beanspruchen die Fälle, in denen epilepsieartige Erscheinungen zum ersten Male im Felde aufgetreten sind. Alle anamnestischen Forschungen haben hier keinerlei Anhaltspunkt dafür gegeben, daß in früherer Zeit schon epileptische Anfälle oder äquivalente Zustände aufgetreten waren. Diese Fälle sind in wissenschaftlicher wie praktischer Hinsicht von gleich großer Wichtigkeit. Sie legen uns die Frage nahe: Sind wir berechtigt hier Neuerkrankungen an Epilepsie im Felde anzunehmen oder handelt es sich lediglich um ganz zufällige Begleiterscheinungen und Äußerungen von andersartigen Krankheitszuständen, wie einige von ihnen oben erwähnt wurden und wie sie von Epilepsie grundsätzlich zu trennen sind? 
Die Beantwortung dieser Frage erfordert eine genaue Untersuchung aller in Betracht kommenden Fälle nach verschiedenen Gesichtspunkten:

1. nach der Vorgeschichte;

2. nach der Entstehung der Anfälle, ihren auslösenden Ursachen und dem klinischen Bilde;

3. nach dem Gesamtverlauf des Leidens.

Die Aufhellung der Vorgeschichte bei derartigen Kranken zeigt uns nicht selten, daß in früheren Jahren, bisweilen schon in der ersten Kindheit, anfallsartige Zustände bestanden hatten, die später ausgeblieben und dem Kranken selbst in Vergessenheit geraten waren. Bei anderen hatte lange Jahre hindurch Enuresis nocturna, nächtliches Aufschrecken, anfallsweises Auftreten von Kopfschmerzen, periodische Reizbarkeit und ähnliche Zustände bestanden, alles Zeichen, die auf eine gewisse Minderwertigkeit der Anlage des Nervensystems schließen lassen. Wieder bei anderen hatten erbliche Belastung oder frühere Kopftraumen vorgelegen. Bonhöffer fand bei 13 einschlägigen Beobachtungen von derartiger ,Feldzugsepilepsie“" durchwegs pathologische Antecedentien.

Bei unseren 36 Beobachtungen ergab die Anamnese in keinem Falle das Bestehen von Anfällen in der früheren Kindheit. In einer Reihe von Fällen wurden diese Angaben der Kranken noch durch zeugenmäßige Erhebungen bei den Angehörigen oder sonstigen Personen in der Heimat oder früheren Arbeitsstätte weiterhin bestätigt. Bei 4 Fällen wurden Angaben gemacht, die auf eine minderwertige Beschaffenheit des Nervensystems schließen ließen. Ein Kranker z. B. litt lange Jahre an Bettnässen, ein anderer an häufig auftretender Übelkeit, besonders nach körperlichen Verletzungen, ein dritter, seit früher Kindheit an nervöser Erregbarkeit, zu der sich nach körperlichen Anstrengungen Anfälle von Atemnot und Schwindel gesellten. Diese beiden letzterwähnten Fälle wird man ohne Bedenken der Gruppe der Psychastheniker (O p penheim) zurechnen müssen. Bei einem vierten Falle war der Krankheitsverlauf folgendermaßen:

28 Jahre alter Unteroffizier, Kraftfahrer. Heredität o. B. Früher nie krank. 1905 Blinddarmentzündung; wegen allgemeiner Körperschwäche nicht gedient. Schon in früherer Zeit nach Überanstrengung wiederholt Schwarzwerden vor den Augen.

1. II. 1915 eingezogen als Kraftfahrer.

11. VII. 1915 ins Feld; hier verantwortungsvolle Posten. Arbeitete den ganzen Tag. In der letzten Zeit verschiedentlich ein Gefühl von allgemeiner Körperschwäche mit Kopfschmerzen und Schwarzwerden vor den Augen; mußte sich sehnell hinsetzen; nach kurzer Erholungspause immer wieder arbeitsfähig.

6. IV. 1916 bei einer Verhandlung auf der Schreibstube plötzlich wieder Übelkeit; konnte sich nicht sogleich setzen, fiel bewußtlos zu Boden. Bericht des beobachtenden Arztes: Bewußtlos, Cyanose des Gesichts, tonisch-klonische Krämpfe, besonders im rechten Arm, Verletzung des Gesichts durch Zerbrechen. des Augenglases, nach 2-3 Minuten wieder klar. 8. IV. 1916 Aufnahme ins Fest. 
Laz. Straßburg. Körperliche Untersuchung vollkommen o. B. Wassermannsche Reaktion im Blute negativ. Während der Lazarettbeobachtung kein Anfall. Entlassung am 3. V. 1916. Wohlbefinden.

Bei weiteren 5 Fällen bestand hereditäre Belastung. Bei 4 Fällen. Alkoholismus, bei einem Fall Lues in der Anamnese. Bei 2 weiteren Fällen hatte Trauma und hereditäre Belastung, bei 2 weiteren Trauma und Alkoholismus vorgelegen. Es bestanden somit in Summe 18 mal $(50 \%)$ Angaben in der Anamnese, die als dispositionelle Ursachen von Bedeutung waren; bei den übrigen 18 fehlte jeder Hinweis.

Von besonderem Interesse waren die Entstehungsbedingungen, die zu den Anfällen geführt hatten. 21 Kranke (58\%) hatten beim Militär gedient; 18 hatten den Dienst mühelos überstanden; bei 3 Kranken waren schon während der aktiven Dienstzeit bisweilen Beschwerden aufgetreten. So gab ein Kranker an, daß er durch körperliche Anstrengungen häufig erregt und von Atemnot befallen worden sei; zwei andere litten während der aktiven Dienstzeit viel an Schwindelanfällen. Ein vierter Kranker gab an, daß er nach Überstehen von Malaria und Skorbut in Südwestafrika während des aktiven Dienstes häufig Schwindelanfälle gehabt habe. Bei allen diesen Fällen ist nachträglich eine genaue Rubrizierung der früheren Krankheitszustände nicht möglich. Der letzterwähnte Fall unterscheidet sich von den vorhergehenden insofern, als die gelegentlichen Schwächezustände nicht infolge einer angeborenen Minderwertigkeit, sondern vielmehr nach postinféktiöser Schädigung des Nervensystems entstanden waren.

Die Einteilung der Fälle nach Altersgruppen war wie folgt:

\begin{tabular}{c||c|c|c|c|c|c}
\hline Jahre & $20-25$ & $25-30$ & $30-35$ & $35-40$ & $40-45$ & 45 u. mehr \\
\hline \hline Fälle & 13 & 8 & 7 & 5 & 3 & - \\
$\%$ & 36,7 & 22,2 & $\mathbf{1 9 , 4}$ & 13,9 & 8,3 & -
\end{tabular}

Bei dem auch nur bedingt statistischen Wert dieser Beobachtung sehen wir doch auch hier wie bei der genuinen Epilepsie ein vorwiegendes Befallensein der ersten 3 Dezennien.

Die Dauer der Kriegsteilnahme, nach der der erste Anfall in Erscheinung trat, war eine sehr verschiedene. In 5 Fällen waren die Anfälle sogleich in der ersten Zeit aufgetreten; in 4 sogar schon in den ersten Tagen der Ausbildungszeit, in dem 5. nach leichter Beschäftigung im Garnisondienst alsbald nach der Einziehung. Von einer schädigenden Einwirkung des Kriegsdienstes kann mithin nicht gesprochen werden. Bei einem anderen Fall trat der erste Krampfanfall nach anstrengendem Turnen auf. Bei den übrigen konnte eine direkte Ursache nicht nachgewiesen werden. 
In 31 Fällen hatte eine durchwegs mehrmonatige Dienstzeit vorgelegen. Die längste Dauer betrug 19 Monate, die kürzeste 4 Wochen. Als Durchschnitt berechnete sich die Dienstzeit im Felde bei diesen 31 Kranken auf 8 Monate. Der militärischen Ausbildung in der Garnison wird keine besondere Bedeutung zuzumessen sein, nachdem sie gut überstanden worden war. Hingegen ist umso mehr die Annahme gerechtfertigt, daß durch die fortgesetzte Anspannung der körperlichen und geistigen Kräfte im Felde sowie sonstige Sehädigungen der Gesundheit Bedingungen geschaffen wurden, die das Auftreten der Anfälle sehr wesentlich begünstigten. Hierfür gibt folgender Fall ein Beispiel:

21 Jahre alter Kanonier.

Heredität: Schwester linkshändig; Pat. war früher Linkser. Schule gut gelernt; seit 1913 aktiv, immer gesund, vertrug alles gut.

2. VIII. 1914 ins Feld.

4. XII. 1914 Schußverletzung im linken Unterarm und Oberschenkel durch Granatsplitter. Damals angeblich bewußtlos. Nach Lazarettbehandlung Anfang März 1915 wieder ins Feld (als Telephonist). Dienst ging an sich gut; Pat. fühlte sich aber infolge starker Gewichtsabnahme sehr kraftlos.

14. VI. 1915 zweite Verletzung durch Gewehrschuß in die rechte Wade.

27. IX. 1915 dritte Verletzung: Quetschung der rechten Hüfte mit Granatsplitterverletzung im rechten Oberschenkel. Gleichzeitig Verschüttung. War längere Zeit bewußtlos; kam erst auf der Sammelstelle wieder zu sich. 4 Tage nach der Verschüttung in der Eisenbahn (Lazarettzug) Anfall mit Zuckungen in den Gliedern und Bewußtlosigkeit; viel Kopfschmerzen; 14 Tage später im Lazarettabort weiterer Anfall. Dezember 1915 beim Ersatzbataillon Anfall. Darnach ins Feld. Nach 14 Tagen im Graben wieder Anfall.

20. V. 1916 (vierte Verletzung) Verschüttung. Darnach 3-4 Anfälle am Tage. Auch in der Folgezeit sehr häufige Anfälle. Oft beim Marschieren Schwarzwerden vor den Augen, Versagen der Beine, Bewußtlosigkeit.

7. V. 1916 nach Lazarett Wiesbaden verbracht; dort häufig Anfälle, einmal Einnässen. Nach 14tägigem Aufenthalt selteneres Auftreten. Von Wiesbaden zum Ersatzbataillon, von dort nach Lazarett 27.

Aufnahme am 7. VIII. 1916. Bisher im Lazarett 1 sicher epileptischer Anfall beobachtet; bei früheren Anfällen häufige Kontusionen des Kopfes. Dauer 5 Minuten. Patient gibt an, er sei wiederholt bis zu 24 Stunden am Scherenfernrohr im Artilleriefeuer tätig gewesen; habe viel Aufregung gehabt; diese sei besonders durch die Furcht vor drohender Falschmeldung bei starkem Trommelfeuer verursacht worden. Entlassung: 23. X. 1916.

Dieser Fall zeigt uns mit Deutlichkeit, wie in offenbarem Fortschreiten mit den Anstrengungen im Felde und den häufigen Schädigungen des Dienstes die Zahl der Anfälle zunahm. Unter dem Einfluß der Lazarettbehandlung ist die Anfallshäufigkeit wieder erheblich zurückgegangen.

Ganz verschiedenartig sind die Angaben der Kranken über eine mutmaßliche veranlassende oder suslösende Ursache der Krampfanfälle. In 21 Fällen konnte keine bestimmte Angabe gemacht werden. Hier war der erste Anfall bei vollkommener Ruhe oder bei leichter Beschäftigung im Unterstande aufgetreten. In 7 anderen Fällen war ein anstrengender 
Marsch oder eine sonstige anstrengende Dienstleistung vorausgegangen. In 2 Fällen trat der erste Anfall auf dem Abort, in 2 Fällen auf einem Patrouillengang, in 2 Fällen nach starker Artilleriebeschießung, in 1 Fall im Schlafe, in 1 Fall auf der Wache auf.

Wiederholt wurde von den Kranken angegeben, daß sie nach der starken Schreckwirkung infolge von Artilleriebeschießung oder Verschüttung viel an Schwindel und allgemeiner Übelkeit gelitten hatten. Einzelne erblickten selbst in den Schwindelanfällen die Vorboten der späteren Krampfanfälle.

Diese Angaben verdienen Beachtung. Sie regen die schon oft gestellte Frage an, in wieweit Schreck überhaupt zur Epilepsie führen kann. Vor 1870 wurden psychische Einwirkungen und unter diesen ganz vorzugsweise der Schreck für eine sehr häufige Gelegenheitsursache der Epilepsie gehalten. Aber schon der Krieg 1870/71 erwies die Unhaltbarkeit dieser Annahme. Auch wenn man die damals zu beobachtenden Fälle weitgehend berücksichtigte, war die Zahl der ,durch Schreck entstandenen Epilespie" gering. Diese Tatsache hat durch den jetzigen Krieg eine neue Bestätigung erfahren. Die schädigende Wirkung des Schrecks auf das Nervensystem soll nicht unterschätzt werden. Aber sie ist doch keineswegs mehr als die eines Gliedes in der Kette der zahlreichen Schädigungen, die ganz allgemein das Nervensystem im Kriege treffen: Schlaflosigkeit, körperliche Anstrengung, unregelmäßige und unzureichende Ernährung, psychische Erregungen and Spannungen. Die Tatsache, daß der Schreck bei vielen Kranken einen besonders lebhaften Dauereindruck hinterläßt, führt sehr häufig dazu, daß alle an den Schreck sich unmittelbar und länger dauernd anschließenden Folgeerscheinungen lediglich der Schreckwirkung zugeschrieben werden. Diese Annahme ist aber nicht berechtigt. Wir werden in jedem Falle weit mehr mit einer Gesamtwirkung der erwähnten sich anhäufenden Schädlichkeiten als mit einfacher Schreckwirkung zu rechnen haben.

Bei nochmaliger Betrachtung obiger Erfahrungen kommen wir zu dem Ergebnis: Eine Anzahl von Feldzugsteilnehmern erkrankt zum ersten Male im Kriege an epilepsieartigen Krankheitserscheinungen. Bei der Hälfte der Fälle finden wir in der Vorgeschichte einzelne krankhafte Anzeichen oder frühere Gesundheitsschädigungen (Traumen), denen nach bisheriger Erfahrung eine gewisse ursächliche Bedeutung zukommt. Nur in einem vierten Teil der Fälle konnte mit der Möglichkeit einer direkt vorausgehenden, im Kriege entstandenen Schädigung als Krankheitsursache gerechnet werden.

Bei den übrigen Fällen blieb die Erforschung der letzteren einstweilen ergebnislos. Es erhebt sich ganz allgemein die Frage: Wie sind diese Krampfzustände klinisch zu bewerten? Ist die Gleichartigkeit ihres Verlaufs, derzufolge wir sie dem Typus der organischen Krampf- 
anfälle zurechnen, von grundsätzlicher Bedeutung, d. h. haben wir ein Recht, in ihrem Auftreten den Hinweis auf das Bestehen eines artgleichen Grundleidens zu erblicken?

Bei genauerer Erforschung der einzelnen Fälle finden wir eine große Mannigfaltigkeit sowohl der einzelnen Anfallsformen als auch ihrer veranlassenden Ursachen.

Bald entstehen schwere Anfälle, bald gleichen die Anfälle den leichteren Typen der bei Epileptikern öfter zu beobachtenden Formen.

Diese Mannigfaltigkeit der einzelnen Formen muß uns vorsichtig machen, nicht ohne weiteres Anfall und Anfall zu identifizieren. Es ist zu bedenken, daß alles, was wir ,organischen Anfall" nennen, sich nur auf eine gewisse Gleichförmigkeit der körperlichen Erscheinungen bezieht, ohne daß wir weiterhin etwas Genaueres über Gleichheit von Ursache und Entstehungsweise auszusagen vermöchten.

Dem Anfall selbst kann somit ausschlaggebende Bedeutung für die Beurteilung des Grundleidens nicht zukommen.

Bei unserem Material handelt es sich um rein klinische Beobachtungen. Es konnte naturgemäß die anatomische Seite nicht berücksichtigt werden. Wir mußten vielmehr lediglich den Ursachen und der Verlaufsweise unser Augenmerk zuwenden.

Bei der Erforschung der Krankheitsursachen haben wir zu unterscheiden:

1. solche, die zur Krankheitsanlage führen;

2. solche, die die Krankheit bei vorhandener Anlage auslösen und offenbar werden lassen.

Bei unseren Beobachtungen ließen sich, wie bereits erwähnt, in der Hälfte der Fälle Angaben auffinden, denen mit einer gewissen Wahrscheinlichkeit eine ursächliche Bedeutung für die Entwicklung der Krankheitsanlage beigemessen werden konnte.

Bei der übrigen Hälfte der Fälle blieb diese Untersuchung ergebnislos.

Fragen wir uns weiter, inwieweit besondere auslösende Krankheitsursachen vorlagen, so ergab sich, daß bei einzelnen Kriegsteilnehmern Anfälle schon kurze Zeit nach der Einstellung und Ausbildung, bei anderen alsbald nach dem Dienst im Felde aufgetreten waren. Bei allen diesen konnte mit hoher Wahrscheinlichkeit eine erhebliche Schädigung der Gesundheit durch den Kriegsdienst ausgeschlossen werden. Aber auch bei zahlreichen anderen, die lange im Felde waren und alle Anstrengungen und Schädigungen des Kriegsdienstes mitgemacht hatten, ließ sich eine bestimmte Ursache für das plötzliche Auftreten des ersten epileptischen Anfalls nicht nachweisen. Der Anfall trat nicht selten nach vorheriger Ruhestellung ein. Bei einzelnen hatte auch an den vorhergehenden Tagen eine nennenswerte Anstrengung oder Gesundheitsschä- 
digung nicht stattgefunden. Bei anderen wurde allerdings die Frage nach einer dem Anfall vorausgehenden Anstrengungen oder Aufregung namentlich durch Artilleriebeschießung bejaht. Hiernach sind die veranlassenden Ursachen in den einzelnen Fällen, wenn nicht fehlend, so doch so verschiedenartig, daß ihnen nicht eine besondere gemeinsame Bedeutung zukommen dürfte. Wir werden demnach um so mehr nach Bedingungen forschen müssen, die in der Eigenart des Trägers liegen. Damit wird die Frage der individuellen Disposition ganz besonders in den Vordergrund gerückt. Abgesehen von vereinzelten Angaben der Vorgeschichte über erbliche Belastung, frühere Traumen oder gelegentliches Versagen gewisser Funktionen des Nervensystems (Bettnässen, gelegentliche Ohnmachten) erfahren wir von den Kranken nichts, was uns weitere Schlüsse gestatten könnte.

Hingegen gibt uns der weitere Verlauf des Zustandes, insbesondere das Wiederauftreten späterer Anfälle einen recht brauchbaren Hinweis auf innere Bedingungen, wie weiter unten noch dargetan wird.

Die vorliegenden Beobachtungen lassen hiernach zwei wesentliche Formen des Krankheitsverlaufs unterscheiden:

1. Fälle mit einmaliger Anfallsepisode;

2. Fälle mit wiederholt auftretenden Anfallsserien.

In 11 Fällen $(28 \%)$ wurde nur 1 Anfall festgestellt; in 25 Fällen (72\%) mehr als 1 Anfall.

Bei den erstgenannten bestanden bei 7 prädispositionelle Ursachen; bei 4 keine.

Bei den letztgenannten bei 9 prädispositionelle Ursachen; bei 16 keine.

Hiernach hatten bei Individuen mit nur einmaligem Anfall verhältnismäßig häufiger prädispositionelle Ursachen vorgelegen. Es scheint. sonach, als ob diese für das Vorkommen wiederholter Anfälle oder Anfallserien keine besondere Bedeutung hätten. Doch muß ejne endgültige Entscheidung dieser Frage einstweilen offen bleiben, da bei den vorliegenden Beobachtungen der Gesamtverlauf nicht genügend überblickt werden kann und mit einem späteren Auftreten von weiteren Anfällen auch bei der ersten Gruppe zu rechnen ist. Wissen wir doch von der genuinen Epilepsie, daß die Zahl der Anfälle außerordentlich wechseln kann; daß auch bei dieser anfangs Anfälle 1-2 mal im Jahre sich zeigen, um dann allmählich häufiger zu werden; daß aber auch wiederum in anderen Fällen große Schwankungen in der Zeit gehäufter Anfallsperioden plötzlich auftreten können.

Bei unseren Fällen mit nur einmal zu beobachtendem epileptischem Anfall sind die Bedingungen zur Annahme einer epileptischen Erkrankung von vornherein am wenigsten erfüllt. Anders bei den Fällen mit gehäuft auftretenden Anfällen. Hier scheint schon die Vielheit der Par- 
oxysmen auf eine erhöhte Krampfdisposition und somit auf eine gewisse Neigung zur chronischen Erkrankungsweise hinzudeuten. Doch sind die einschlägigen Fälle keineswegs alle gleichartig beschaffen. In den einen finden wir ein langedauernd anfallsfreies Intervall, in den anderen serienweise schnell aufeinanderfolgende Krampfanfälle, nur durch kurze Zeitabschnitte getrennt. Der krankhafte Vorgang, der den Kramapfzustand entstehen läßt und zur Auslösung bringt, erreicht bei den erstgenannten Fällen seine Reizschwelle viel langsamer als bei den letzteren. Vielleicht haben wir es bei diesen mit der immer noch anhaltenden Nachwirkung des erstmalig besonders intensiven Krampfreizes zu tun, die eine Zeitlang immer noch zu weiteren Entladungen führt. Wir wissen allerdings nichts Näheres über die Ursachen derartiger Verschiedenheiten. Doch darf im Hinblick auf gewisse klinische Erfahrungen bei anderen Krankheitszuständen (Spasmophilie, Eklampsie) mit gewissem Recht angenommen werden, daß eine einmalig zu beobachtende Serie von Krampfanfällen nach ihrer Genese und klinischen Natur anders zu beurteilen sind als die durch lange Zeitabschnitte getrennten. Die Erstgenannten sind vergleichbar mit den Krampfformen, die $\mathrm{KuBmaul \text {, }}$ Tenner und Nothnagel nach Verblutung an Tieren beobachtet und als anämische Krämpfe bezeichnet haben. Sie haben bis in die neueste Zeit das Interesse der Epilepsieforscher in Anspruch genommen; ihre Deutung ist vielfach variiert worden. Ganz ähnliche Krampfformen sind bei Störungen der Zirkulation und bei Vergiftungszuständen (Erhängten, Kohlenoxydvergiftungen) beobachtet worden. Bei allen diesen Fällen beobachten wir den pathologischen Vorgang eines einzelnen oder einer Serie von Anfällen. Diese ,,anämischen Krämpfe" gaben Veranlassung zu der sog. medullären Theorie der Epilepsie. Hiernach sollte der einzelne epileptische Anfall primo loco durch Exregung der vasomotorischen Centren in der Medulla oblongata, durch diese wiederum eine Anämie in der Hirnrinde erzeugt werden, wodurch schließlich der Anfall ausgelöst wird. Die medulläre Theorie ist heute mehr und mehr verlassen worden. Wir wissen jetzt, daß die sog. anämischen Krämpfe durchaus nicht einem epileptischen Anfall entsprechen, sondern höchstens daran erinnern. Auch die von uns beobachteten Fälle der Feldzugepilepsie sind wir in keiner Weise berechtigt nach ihrem episodenhaften Auftreten der Epilespie zuzurechnen. Diese Annahme hat durch den weiteren Verlauf eine Bestätigung erfahren. Wenn nach langdauernder Beobachtung weiterhin für derartigen Individuen keine Krämpfe wieder auftreten, können wir mit Sicherheit annehmen, daß eine Neigung zu dauerndər Krampfreaktion und damit die Gefahr der Entwicklung eines chronischen Leidens nicht besteht. Dort, wo wir die Anzeichen einer früheren zeitweisen Erschöpflichkeit des Nervensystems (Schwindelanfälle, Ohnmachten) in der Anamnese finden, werden wir annehmen 
können, daß es sich um asthenische oder affektepileptische Anfälle (Bratz) handelt. Diese Annahme besteht auch in den Fällen zu Recht, wo eine dem Anfall unmittelbar vorausgehende Ursache nicht auffindbar ist. Wissen wir doch, daß solche Anfälle unvermittelt und gehäuft auftreten können, ohne daß ein affektiver Ursprung vorhanden ist (Binswanger). Letzterer kann im Kriege durch mannigfache andersartige Schädigungen reichlich Ersatz finden. Zustände von cerebraler Erschöpfung im Gefolge von Zirkulationsschwäche oder Inanition, chronisch-anämische Erscheinungen oder auch geistige Überanstrengung können bei von Hause aus widerstandsschwachen Individuen das Auftreten von Anfällen begünstigen, in ähnlicher Weise wie wir es bei den sog. affektepileptischen bzw. psychasthenischen Formen von der Friedenszeit her kennen. Wenn wir unmittelbare krampfauslösende Ursachen in vielen Fällen nicht nachweisen können, so ist dies nicht verwunderlich angesichts der Schwierigkeit, unter den zahlreichen allgemeinen Ge. sundheitsschädigungen eine bestimmte namhaft zu machen. Ebenso darf der mangelnde Nachweis von degenerativ-psychopathischer Veranlagung (Oppenheim. Bratz) nach der Anamnese uns nicht irre machen in der oben erwähnten Deutung und Rubrizierung der Anfälle. Sehen wir doch auch in diesem Kriege neurotische, insbesondere hysterisehe Krankheitszustände der mannigfachsten Art bei Individuen auftreten, die früher keinerlei nervöse Krankheitserscheinungen geboten hatten.

Wenn wir somit den serienweise auftretenden Anfällen eine Bedeutung im Sinne von Epilepsie nicht beimessen können, so ist dies noch weit weniger berechtigt in den Fällen, bei denen überhaupt nur ein einziger Anfall zur Beobachtung kam. Hier ist die Versuchung besonders groß, nach einer andersartigen Ursache zu forschen, als wir für Epilepsie und alles damit Verwandte anzunehmen geneigt sind. Als besonders naheliegend käme der Hitzschlag in Frage. Aber wir finden bei diesem in der Regel körperliche Anstrengungen und Wärmestauungen vorausgegangen; dies trifft für keinen unserer Fälle zu. Auch bei Spasmophilie sehen wir nicht selten ähnliche Anfälle wie bei den hier aufgeführten Kranken; nur fehlt hier die spasmophile Diathese, die eine solche Erklärung rechtfertigen könnte. $\mathrm{Zu}$ bemerken ist allerdings, daß es auch Fälle von Krämpfen in kindlichem Alter gibt, ohne daß eine spasmophile Grundlage vorhanden zu sein braucht. Wir sehen hier nach zufälligen Reizen Konvulsionen auftreten, sei es in Form eines einzigen Anfalles oder in einer Serie von Anfällen, ohne das Epilepsie sich anschließt. Genaueres vermögen wir bisher über die Ursache derartiger Anfälle nicht zu sagen.

Unter nochmaliger Berücksichtigung aller obigen Erwägungen kommen wir zum Schluß: 
Bei einem Teil der sog. Feldzugepilepsie spielt für die Entstehung der Anfälle der Krieg nur eine untergeordnete Rolle. Hier hatten erbliche Belastung, frühere Traumen, oder auch ein gelegentliches Versagen einzelner Funktionen des Nervensystems vorgelegen, alles anamnestische Angaben, die auf eine bereits bestehende Schwäche und Krankheitsbereitschaft des Nervensystems hindeuten. Der Krieg und seine Schädigungen kommen nur als verschlimmernde oder auslösende Ursachen in Betracht. Diese Fälle gehören zur Gruppe der affektepileptischen bzw. asthenischen Anfälle.

Bei einem anderen Teil der sog. Feldzugepilepsie vermissen wir Antecedentien, die eine Deutung im ebenerwähnten Sinne zulassen. Trotzdem sind wir auch hier nicht berechtigt, Epilepsie anzunehmen. Nach dem episodenhaften Auftreten haben wir auch bei diesen Kranken anzunehmen, daß diese Anfälle den affektepileptischen nahe verwandt, wenn nicht mit ihnen identisch sind. Sie unterscheiden sich von diesen nur insofern, als anamnestische Anhaltspunkte, im Sinne von Affektepilepsie vermißt werden. Aber die Annahme erscheint gerechtfertigt, daß hier die Anstrengungen und Schädigungen des Krieges eine allgemeine Schwächung des Nervensystems bewirkt und damit ganz wesentlich mit zu dem Auftreten dieser Anfälle beigetragen haben.

Eine weitere Fra,ge muß einstweilen ungelöst bleiben, ob bei diesen Fällen nicht eine besondere Krampfbereitschaft des Gehirns vorliegt. Ein Vergleich mit anderen Beobachtungen, insbesondere den Epilepsieformen, die nach Schußverletzungen des Gehirns auftreten, muß uns dies nahelegen. Sehen wir doch, daß auch hiervon nur immer ein verhältnismäßig geringer Teil an traumatischer Epilepsie erkrankt. Diese Tatsache läßt auch bei diesen Fällen die Annahme einer besonderen Krampfbereitschaft des Gehirns gerechtfertigt erscheinen.

Nur ein sehr geringer Teil der nicht durch Trauma entstandenen Fälle darf mit Recht der Epilepsie zugeteilt werden.

Unsere Beobachtungen enthalten zwei hier anzuführende Beispiele:

1. Karl B., 26 Jahre, Bergmann, Kanonier.

Heredität o. B. Früher nie kxank. Gut in der Schule gelernt; seit 1908 Bergmann. 1911 gedient, ohne Beschwerden. 3. VIII. 1914 mobil; 9. VIIr. 1914 ins Feld.

4. VIII. 1915 plötzlich ohne äußeren erkennbaren Anlaß vom Pferd gefallen, wurde einige Meter mitgeschleift, habe mit Händen und Füßen um sich geschlagen.

11. VIII. bis 21. IX. im Kriegslazarett Gent; von dort als felddienstfähig entlassen.

22. IX. nach 8 tägigem Dienst wieder Anfall in der Nacht. 1. X. 1915 weiterer Anfall. Bis 29. I. 1916 im Heimatslazarett, dort alle 14 Tage Anfälle.

30. I. 1916 Aufnahme im Fest.-Laz. 27.

1. II. 19164 typische epileptische Anfälle innerhalb 2 Tagen.

11. II. 19163 epileptische Anfälle mit Zungenbiß beobachtet.

24. II. 1916 als d. u. entlassen. 
In diesem Falle werden wir mit großer Wahrscheinlichlkeit annehmen können, daß der Sturz rom Pferde bereits in einem epileptischen Anfalle erfolgte.

2. Fall (36). Richard K., 29 Jahre, Förster, Oberjäger. Heredität ausdrücklich o. B. Als Kind gesund, in Schule gut gelernt. 1906-1908 gedient, nie krank. $3 / 4$ Jahre vor der Mobilmachung bisweilen Kopfweh mit Schwindelgefühl und allgemeiner Mattigkeit.

3. VIII. 1914 mobil. September 1914 nach Westen. Nach 8tägigem Liegen in Stellung eines Nachmittags starke Anstrengung bei $21 / 2$ ständigem unvnterbrochenem Schießen. Nach Ruhelage im Unterstand plötzlicher Anfall mit Zungenbiß und Hautabschürfung; darnach viel Kopfweh und Mattigkeit. 2 Tage Schonung, dann wieder Dienst. Auf Märschen oft Schwindelanfälle mit ,aufsteigendem Gefühl in der Kehle".

4 Wochen später vor Ypern 2 Anfälle.

9. 11. 1914 Gewehrschuß durch den linken Unterarm. 4 Wochen im Lazarett Wetzlar; dort 3 Anfälle. Von März 1915 bis August 1916 anfallsfrei. Der Dienst fiel ihm leicht, doch bei Märschen oft Schwindelgefühl.

20. VIII. 1916 nach 4stündigem Exerzierunterricht plötzlicher Anfall mit Zungenbiß und Kopfverletzung.

6. IX. 1918 wieder Anfall mit Zungenbiß und Gesichtsverletzung.

6. IX. 1916 Aufnahme ins Lazarett 27. Deutliche Reste der frischen Gesichtsverletzung. Körperlicher Befund o. B. Wassermannsche Reaktion im Blute negativ. Mitte Oktober im Lazarett plötzlicher absenceartiger Schwindelanfall; sonst kein Anfall bisher beobachtet.

Hier geben uns anamnestische Forschungen über frühere Anfälle oder anfallsartige Zustände bei dem erstgenannten der beiden Fälle keinerlei Anhaltspunkte. Bei dem 2. Fall wird uns berichtet, daß bereits vor dem Kriege bisweilen Kopfweh mit Schwindelanfällen und allgemeiner Mattigkeit bestand. Somit ist auch in diesem Falle die Möglichkeit, daß das Leiden schon vor Kriegsausbruch seinen ersten Anfang genommen hatte, nicht sicher auszuschließen.

Was aber beide Fälle mit hoher Wahrscheinlichkeit der Epilepsie zuzuteilen berechtigt, ist die ausgesprochene epileptische Art der Anfälle und ihr fortgesetzt intermittierendes Auftreten.

Unter Berücksichtigung der Erfahrungen aus der Friedenszeit ist erstmaliges Auftreten von Epilepsie im 2. bis 3. Dezennium, wenn auch schon seltener, so doch immerhin vorkommend.

Wir werden daher auch für diese Fälle von im Kriege entstandener Epilepsie annehmen können, daß das Auftreten des Leidens im Kriege ein mehr zufälliges Ereignis ist, mag auch immerhin ein gewisser Grad von Gesundheitsschädigung durch Kriegsstrapazen als krankheitauslösender Faktor in Betracht kommen.

\section{Tabelle A.}

Enthält 152 Fälle von Epilepsie, die schon vor Kriegsausbruch bestanden hat. Von ihrer Veröffentlichung wird an dieser Stelle aus äußeren Gründen Abstand genommen. 


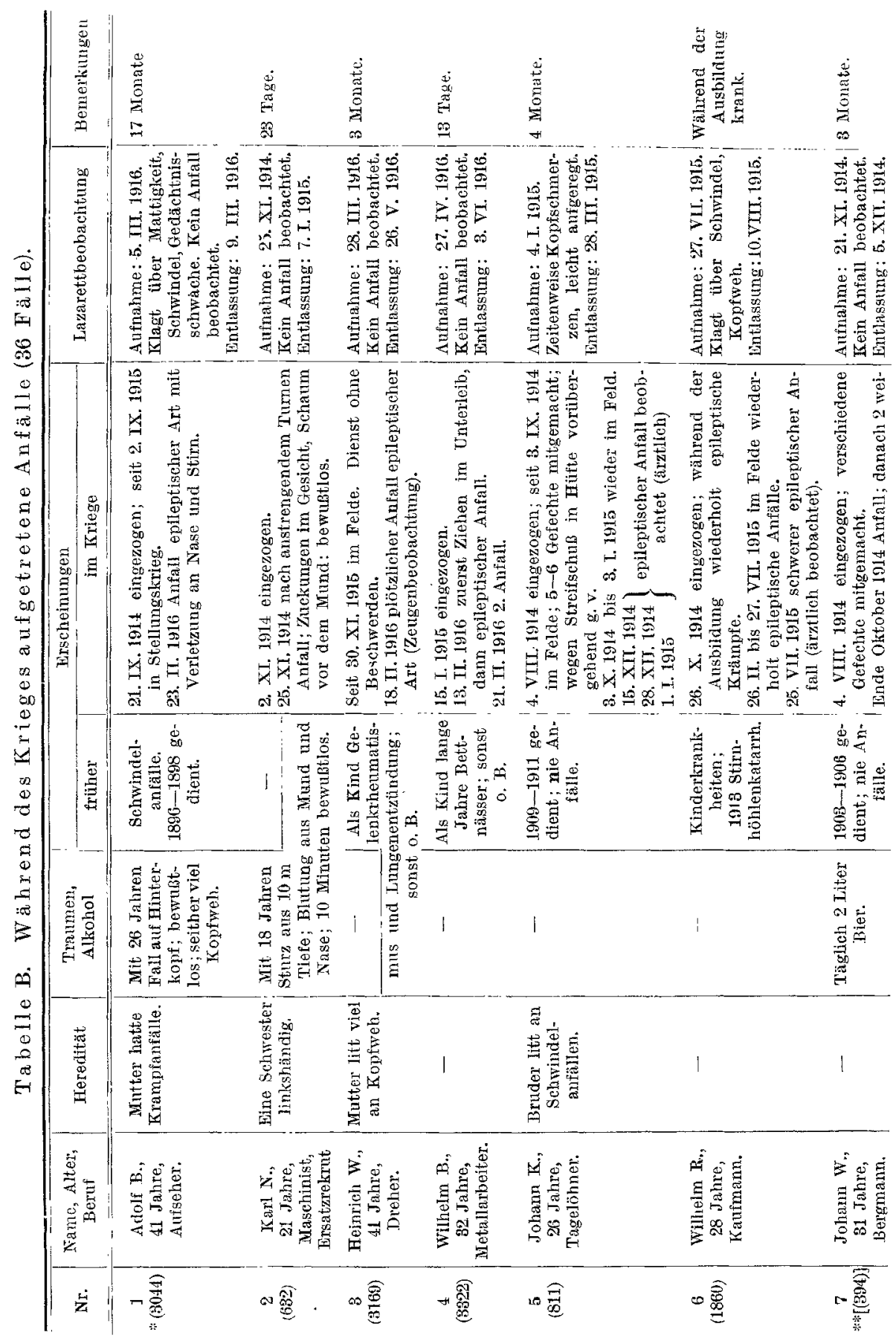


Epilepsie im Kriegre.

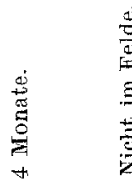

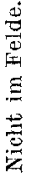

节

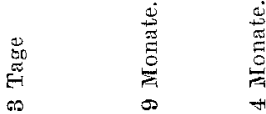

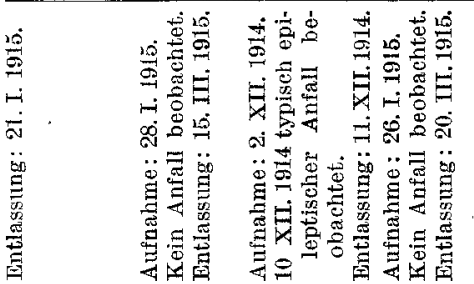

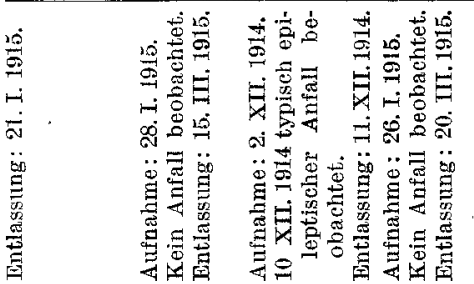

+

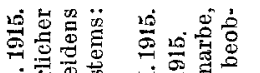

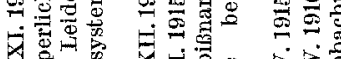

जी:

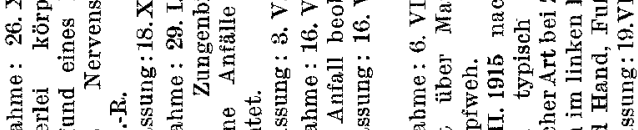

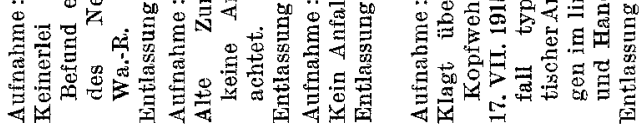

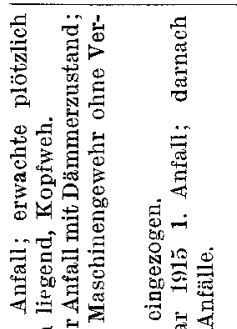

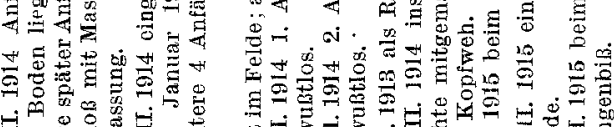

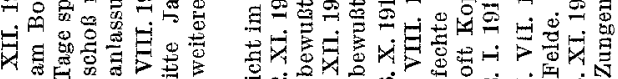

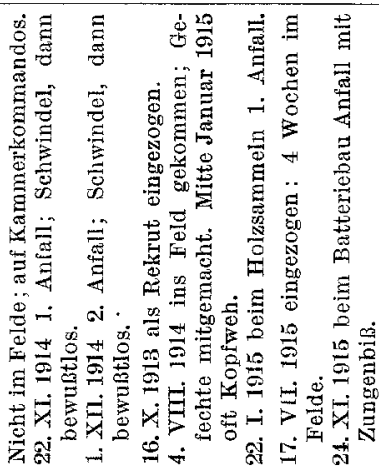

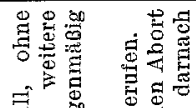

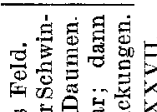

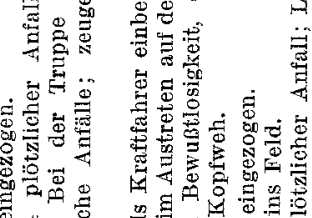

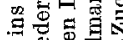

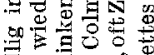

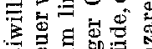

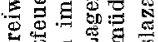
iी

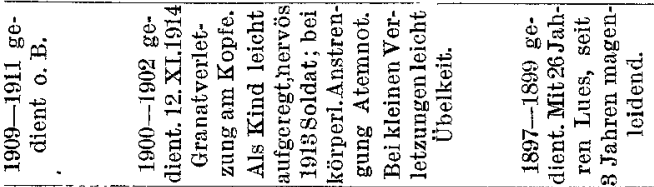

क人

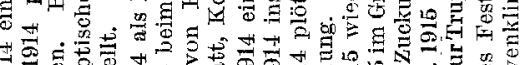

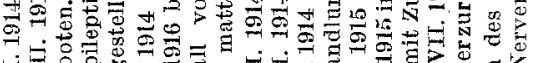

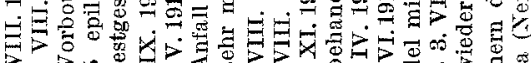
$P=0$ w

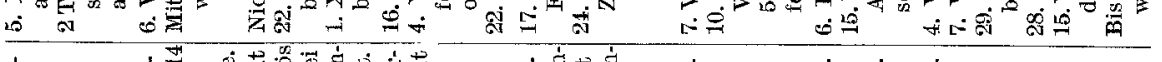

空

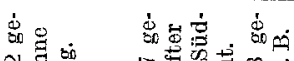

क्ञ

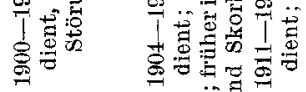

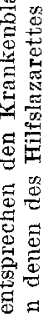

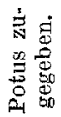

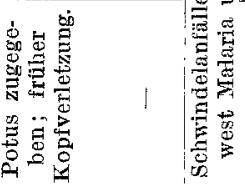

\begin{tabular}{|c|c|c|c|c|c|c|c|}
\hline 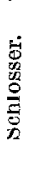 & 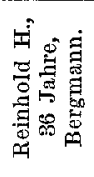 & 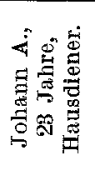 & 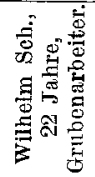 & 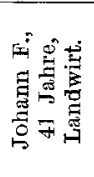 & 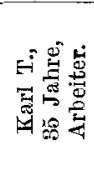 & 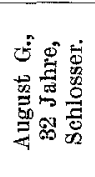 & 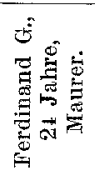 \\
\hline & $\sigma \stackrel{\text { 总 }}{3}$ & 웡 & $7 \overrightarrow{\hat{0}}$ & 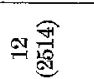 & $m \widehat{s}$ & 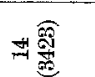 & 10 \\
\hline
\end{tabular}




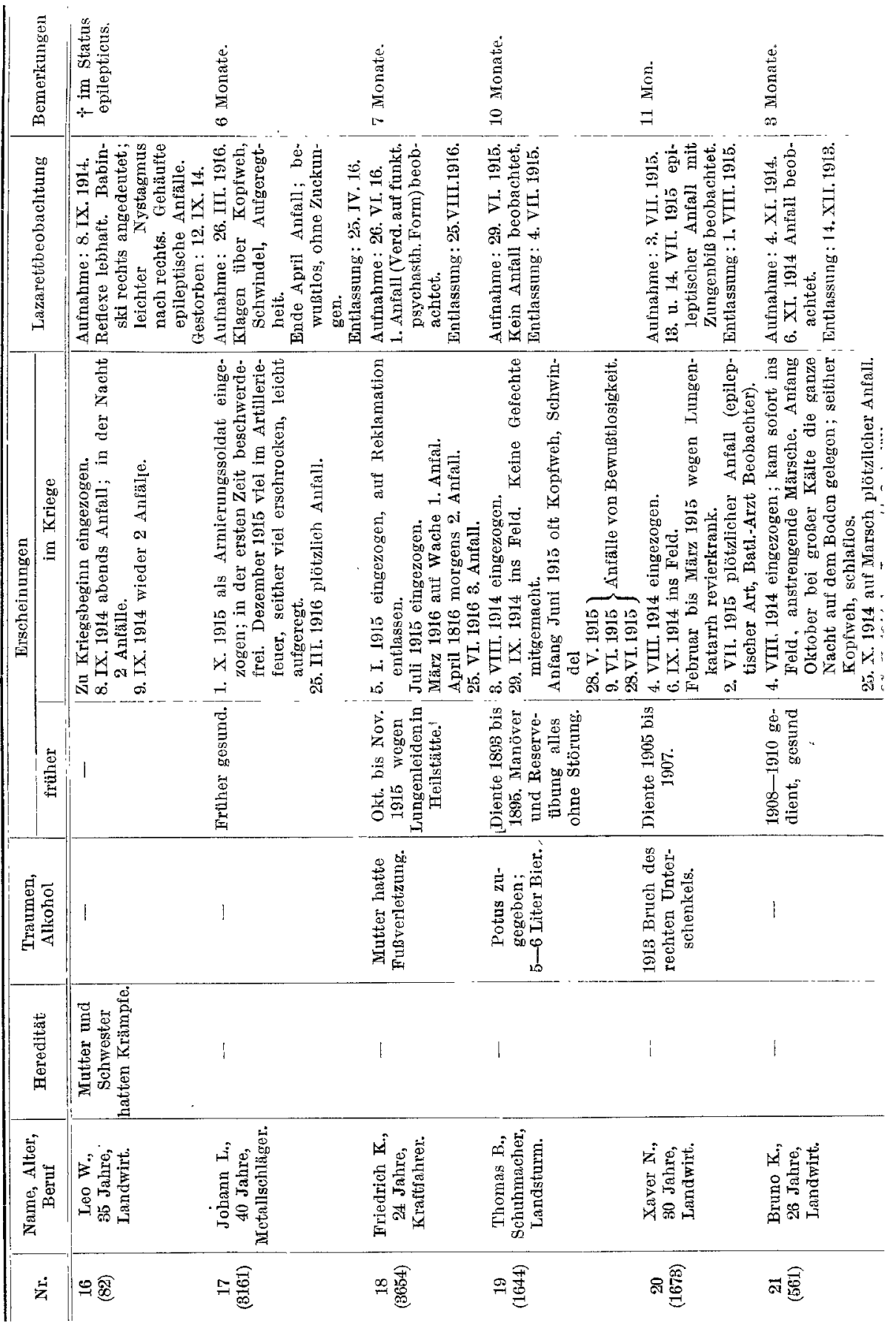




\begin{tabular}{|c|c|c|c|c|c|}
\hline & 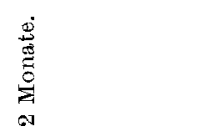 & 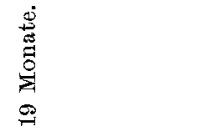 & 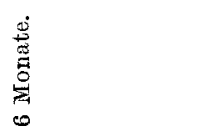 & 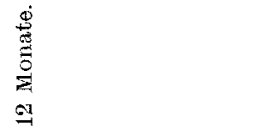 & 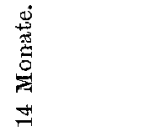 \\
\hline 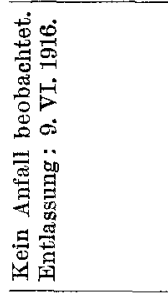 & 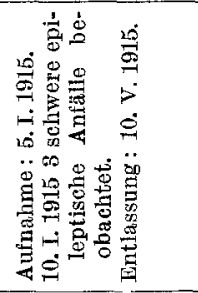 & 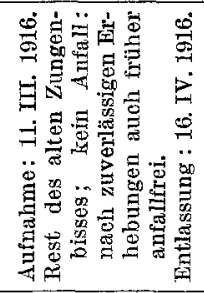 & 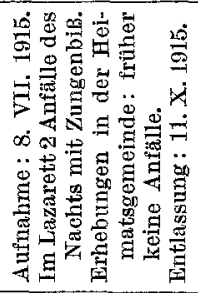 & 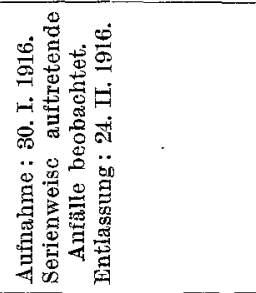 & 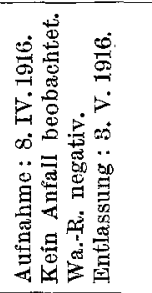 \\
\hline 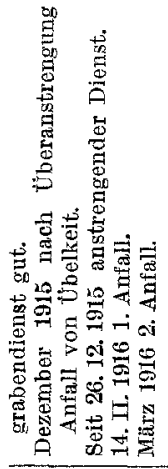 & 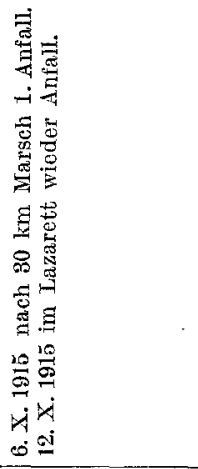 & 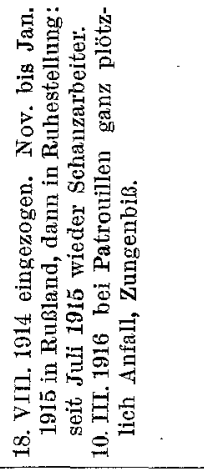 & 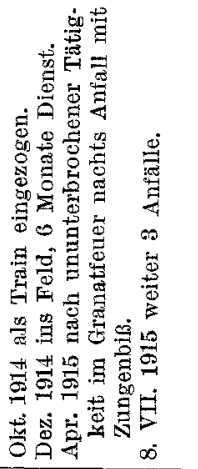 & 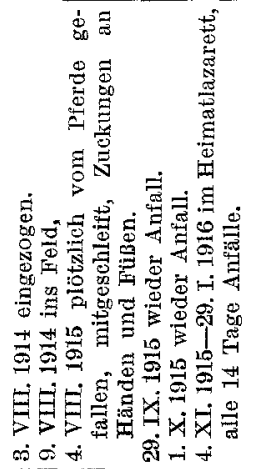 & 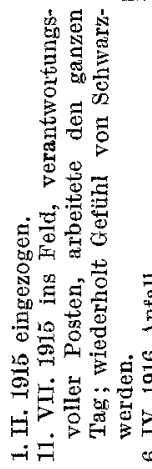 \\
\hline \multirow[t]{2}{*}{ 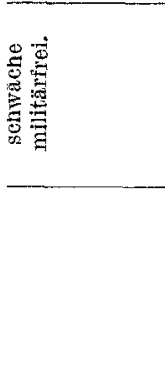 } & 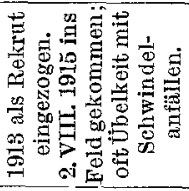 & 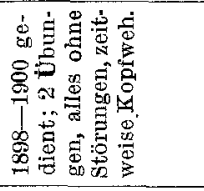 & 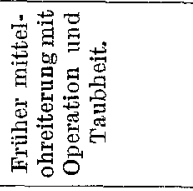 & 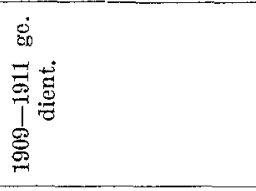 & 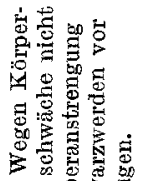 \\
\hline & 1 & 1 & 1 & $i$ & 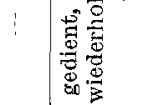 \\
\hline
\end{tabular}

\begin{tabular}{|c|c|c|c|c|c|}
\hline 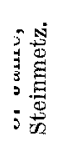 & 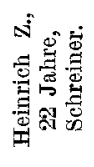 & 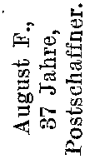 & 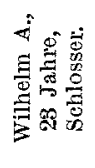 & 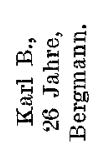 & 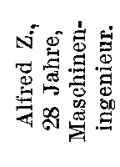 \\
\hline & $\stackrel{\widehat{a}}{\alpha}$ & 可害 & 월용 & 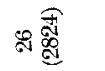 & 品㩊 \\
\hline
\end{tabular}




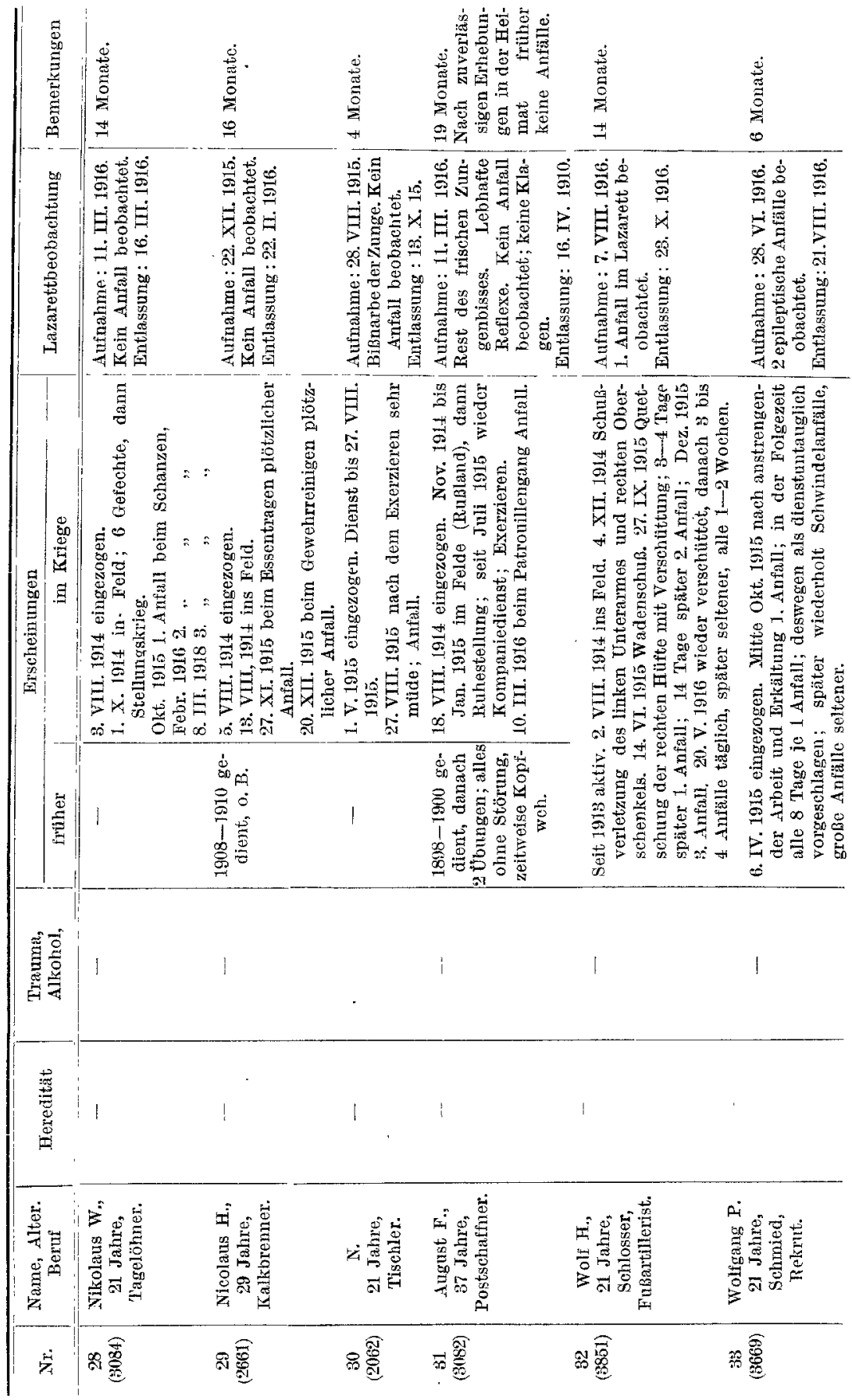




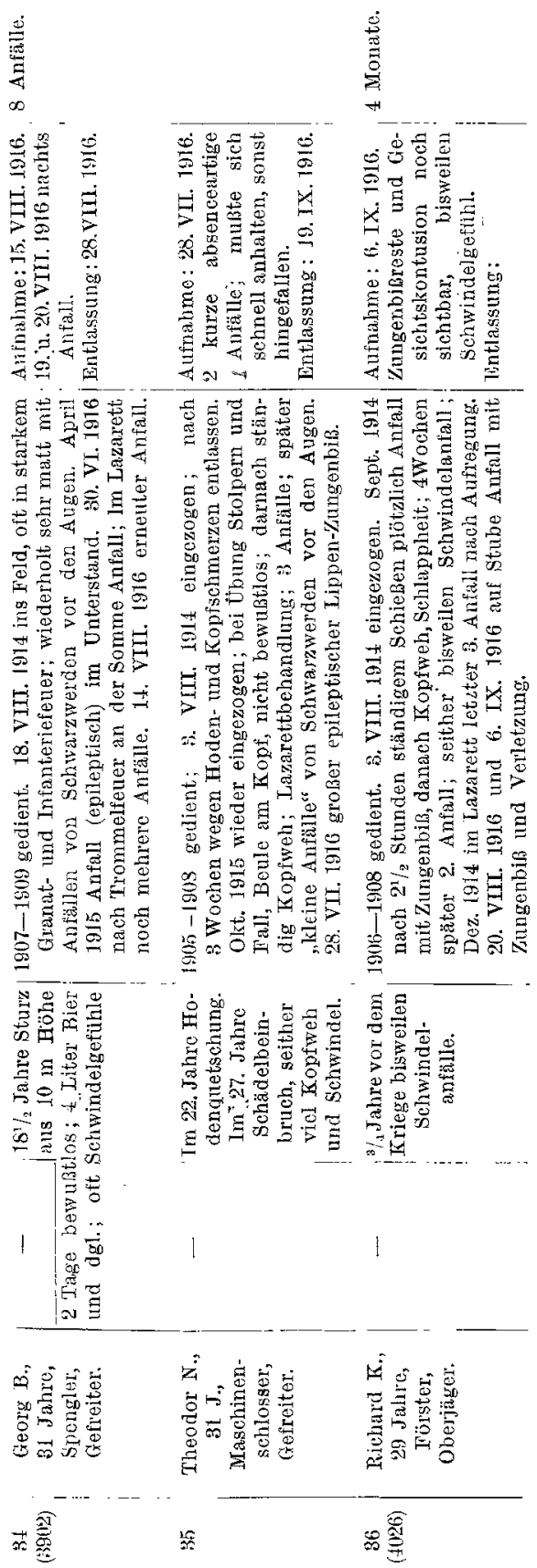




\section{Literatur.}

1) Bonhöffer, Erfahrungen über Epilepsie und Verwandtes im Feldzuge, Monatsschr. f. Psychiatr. u. Neurol. 38, H. 1/2. - ?) Bratz, Die affektepileptischen Anfälle der Neuropathen und Psychopathen. Monatsschr. f. Psychiatr. u. Neurol. 29. - ${ }^{3}$ ) Bruns, Beitr. z. klin. Chirurg. 101. - $\left.{ }^{4}\right)$ Bruns, Kramer, Ziehen, Lehrbuch der: Nervenkrankheiten des Kindesalters. - ${ }^{5}$ ) Gruhle, Über die Fortschritte in der Erkenntnis der Epilepsie in den letzten 10 Jahren und über das Wesen der Krankheit. Zeitschr. f. d. ges. Neurol. u. Psychiatr. 2, H. I. 1910. $\left.{ }^{6}\right)$ Kraepelin, Lehrbuch der Psychiatrie. - 7) Oppenheim, Über psychasthenisehe Krämpfe. Journ. f. Psych. u. Neurol. 1905, S. 247; Lehrbuch der Nervenkrankheiten. - ${ }^{8}$ ) Westphal, Agoraphobie. Areh. f. Psychiatr. 3. 1871; Über eigentümliche mit Einschlafen verbundene Anfälle. Arch. f. Psychiatr. \%. 9) Sanitätsbericht über die deutschen Heere 1870/71. Bd. ร. IV. Med. Teil. B. VIII. Kapitel, S. 290. 